\title{
THÉMATISATION DES COMPLÉMENTS CIRCONSTANCIELS
}

\author{
Gaston GRoss \\ Université Paris 13
}

\begin{abstract}
This study aims at accounting for one of the properties of circumstantial complements which has been unjustly neglected in linguistic studies, i.e. their capacity to be thematized as subjects of a main verb. This reconfiguration mobilizes a certain number of appropriate verbs which cannot be described without reference to this phenomenon. The article then focuses on complements of place, time, cause, goal, condition, concession as well as on certain qualitative constructions.
\end{abstract}

\section{INTRODUCTION}

Nous allons examiner, dans ce qui suit, certains types de restructurations qui permettent de transformer des compléments circonstanciels en sujets. On considère en général que ces modifications ne concernent que les arguments du premier ordre, en particulier les objets directs, qui peuvent figurer en position de sujet en cas de passivation. La thématisation s'observe aussi avec la plupart des compléments circonstanciels. Cela induit certains procédés qui n'ont guère été étudiés jusqu'à présent. En particulier, il ne faut pas confondre la thématisation avec la mobilité de ces compléments qui peuvent figurer au début comme à la fin des phrases. Pour des raisons de clarté, nous allons d'abord rappeler les propriétés des compléments dits circonstanciels, nous proposerons ensuite une analyse des divers moyens qui sont en oeuvre dans ces restructurations. 


\section{DÉFINITION DES COMPLÉMENTS CIRCONSTANCIELS}

Nous nous inspirons de la Grammaire méthodique du français (Riegel, Pellat, Rioul, 1994 : 140-145). Ces auteurs attribuent trois propriétés à ce type de compléments :

- ils sont facultatifs

- ils se démultiplient librement

- ils sont mobiles dans les limites de la phrase entière

Ces auteurs mettent en outre l'accent sur diverses propriétés de ce type de compléments.

La mobilité est la propriété vraiment caractéristique du complément circonstanciel. Cette propriété s'explique par son statut syntaxique de constituant périphérique. Comme constituant immédiat de la phrase, il ne dépend pas d'un autre syntagme et en particulier n'est pas régi par le verbe. Il ne fait pas partie de la valence verbale.

Le complément circonstanciel est le plus souvent un groupe prépositionnel dont la préposition spécifie le type de rapport qui l'unit au reste de la phrase : temporel (avant six heures), spatial (au bout du chemin), causal (à cause de mon lumbago), concessif (malgré les avertissements répétés).

La fonction de complément circonstanciel peut aussi être remplie par une subordonnée circonstancielle (Je suis venu pour que vous ne soyez pas seul) ou réduite à une construction infinitive (Je suis venu pour m 'inscrire) ou par une participiale (le chat parti, les souris dansent).

Il y a identification des subordonnées aux compléments circonstanciels : Prép $\mathrm{N}=$ Prép que $\mathrm{P}:$ par avarice $=$ parce qu'il est avare .

À partir de ces constatations, l'objet de cet article est de mettre en évidence une propriété des compléments circonstanciels qui est généralement négligée : ils sont susceptibles d'être thématisés, c'est-à-dire figurer en position de sujet d'une phrase dite principale. Nous examinerons le phénomène successivement à propos des compléments de lieu, de temps, de cause, de but, de condition et de concession.

\section{COMPLÉMENTS DE LIEU}

Nous passerons en revue certains types de compléments de lieu. Nous les décrivons en termes de classes d'objets, pour que l'analyse soit plus précise. 


\subsection{Relations de CONTENANT À CONTENU}

La préposition dans permet, parmi d'autres moyens linguistiques, de traduire la relation établie entre un contenant et un contenu, comme dans l'exemple suivant : Il y a cinq litres dans ce bidon. Le complément en dans $N$ est déplaçable : Dans ce bidon, il y a cinq litres. Il se peut que la valeur informative de ces deux phrases ne soit pas identique, même si les éléments lexicaux gardent leurs propriétés syntaxiques. Si le complément circonstanciel se trouve au début de la phrase, il est en quelque sorte topicalisé, mais il reste un complément. Imaginons qu'à valeur sémantique constante entre les différents éléments de cette phrase, nous voulions mettre le mot bidon en position de sujet. La langue met alors à notre disposition le verbe contenir : Il y a cinq litres dans ce bidon; Ce bidon contient cinq litres. Observons, au passage, que ce verbe, apparemment transitif direct, n'a pas de forme passive : *Cinq litres sont contenus dans ce bidon.

D'autres verbes sont en mesure de jouer le même rôle de thématisation d'un circonstanciel de lieu dans des emplois métaphoriques :

Il y a plusieurs erreurs dans cette traduction

Dans cette traduction, il y a plusieurs erreurs

Cette traduction (comprend, comporte) plusieurs erreurs

Il y a un trop grand nombre de handicaps dans cette région

Dans cette région, il y a un trop grand nombre de handicaps

Cette région concentre un trop grand nombre de handicaps

Voici d'autres verbes jouant un rôle syntaxique similaire : englober, retenir, réunir, être farci de, être truffé de, etc. :

Il y avait un grand nombre de fautes dans cette rédaction

Cette rédaction était truffée de fautes

Il y a toutes les matières scientifiques au programme de terminale

Le programme de terminale englobe toutes les matières scientifiques

Cette classe comprend un assez grand nombre d'équivalents lexicaux de l'expression il y a dans, à savoir : avoir, comporter, comprendre, compter, concentrer, embrasser, emprisonner, enchaîner, enclore, enfermer, englober, enserrer, être composé de, inclure, jauger, mesurer, posséder, présenter, receler, recevoir, renfermer, retenir, tenir. La même relation peut être traduite par des adjectifs comme : être rempli de. On aura alors les équivalents lexicaux suivants : 
bondé, bourré, chargé, comblé, débordant, farci, garni, gavé, gonflé, gorgé, hérissé, muni, pénétré, pétri, peuplé, plein, saturé, truffé. Les antonymes sont les suivants : exempt, vacant, vide, etc.

\subsection{COMPLÉMENTS SCÉNIQUES}

Nous définissons ces compléments comme des lieux dans lesquels se déroule un événement. Ce complément est différent de celui qui traduit la localisation d'un objet, comme dans : Il y avait un livre sur la table, où il y a peut être paraphrasé par se trouver : Un livre se trouvait sur la table. Nous prenons en considération des phrases du type : Il y a eu un terrible accident à Paris; $\grave{A}$ Paris, il y a eu un terrible accident et nous essayons de mettre le locatif Paris en position de sujet. Un des moyens linguistiques toujours possibles consiste à introduire un substantif classifieur comme lieu : Paris est le lieu où il y a eu un terrible accident. Mais cette phrase est très redondante. Une autre restructuration est beaucoup plus naturelle. Celle-ci se fonde sur une métaphore mettant en jeu certains $<$ lieux de spectacles> :

Paris a été le théâtre d'un terrible accident

La chambre des députés a été la scène d'un coup d'État manqué

Ce château a été le décor de fêtes somptueuses

Ces possibilités métaphoriques sont cependant limitées :

*Cette rue a été le cinéma d'un crime abominable

*Cet endroit a été les planches de l'assassinat du duc de Guise

On trouve encore des mots comme site :

Reggane a été le site de la première explosion nucléaire française

\subsection{LOCATIFS APPROPRIÉS}

Comme nous venons de le voir, les éléments prédicatifs qui permettent à un locatif de passer en position de sujet sont fonction de la nature sémantique des compléments de lieu et de leur corrélat. Ainsi les instruments de mesure ont-ils des verbes qui leur sont propres : 
Il est 3 heures à cette montre

À cette montre, il est 3 heures

Cette montre affiche 3 heures

\subsection{COMPléMENTS DE « SURFACE »}

Il existe un certain nombre de substantifs qui désignent des traces observables sur une surface : égratignure, marque, rayure, etc. :

Il y a des traces de coups sur son visage

Il y a une série de rayures sur la table

C'est le verbe porter qui permet à ce type de lieux de passer en position de sujets :

Son visage porte des traces de coups

La table porte une série de rayures

On peut considérer comme synonymes de cet emploi de porter des verbes comme : afficher, arborer, avoir, exhiber, manifester, montrer, offrir, posséder, présenter.

\section{Compléments de TeMps}

Le phénomène s'observe aussi avec les compléments de temps. Ceux-ci ont la même liberté syntaxique que les locatifs. Comme pour le lieu, il existe des classifieurs de temps qui permettent la mise en évidence du complément temporel :

Paul a choisi de partir au moment des élections

Les élections sont le moment que Paul a choisi pour partir

Les bons seront récompensés un jour

Le jour arrivera où les bons seront récompensés

Nous dûmes partir le lendemain

Le lendemain est le jour où nous dûmes partir

Certains verbes, comme connaître, permettent à un complément de temps de devenir sujet : 
Il y a eu des révoltes sanglantes au moyen âge

Le moyen âge a connu des révoltes sanglantes

En position d'objets, il y a deux sortes de compléments de temps : ceux qui sont bornés à droite, c'est-à-dire qui désignent des accomplis et ceux où le terme de l'action n'est pas nettement indiqué. Les compléments sont respectivement en $N$ et pendant $N$ :

Paul finira ce travail en trois mois

En trois mois Paul aura fini ce travail

Notons que les compléments de temps ont cette particularité qu'ils peuvent, par restructuration, figurer en position de complément d'objet. On utilise à cet effet le verbe mettre :

Paul a mis trois mois (à, pour) finir ce travail

Dans l'autre exemple, c'est le verbe passer qui a la même fonction :

J'ai réparé le filet pendant toute la nuit

Pendant toute la nuit, j'ai réparé le filet

J'ai passé toute la nuit à réparer le filet

Un certain nombre d'autres verbes ont le même rôle syntaxique avec, dans certains cas, des modalités spécifiques :

- suffire implique un sujet :

J'ai réussi à boucler ce travail en deux heures

Deux heures m'ont suffi pour boucler ce travail

- consacrer implique un objet :

J'ai corrigé ce texte pendant la matinée

J'ai consacré la matinée à corriger ce texte

On notera encore des verbes comme :

- réserver :

À l'avenir nous aurons beaucoup de surprises

L'avenir nous réserve beaucoup de surprises 
- débuter :

L'année civile commence au premier janvier

Le premier janvier débute l'année civile

La mise en évidence d'un complément de temps peut s'opérer à l'aide d'autres moyens linguistiques :

Les hommes mouraient de faim à une certaine époque

À une certaine époque les hommes mouraient de faim

Il fut une époque où les hommes mouraient de faim

D'autres mises en évidence ne conservent pas aussi nettement le parallélisme entre les deux phrases :

Je n'ai pu finir la conclusion, faute de temps

Le temps m'a fait défaut pour finir la conclusion

On ne peut plus actuellement répondre de la sorte

Le temps (n'est plus, est révolu) où l'on pouvait répondre de la sorte

Paul a quitté le pays depuis longtemps

Le temps a passé depuis que Paul a quitté le pays

Il faut répondre sérieusement maintenant

Le moment est venu de répondre sérieusement

Il attendait toujours minuit pour aller au restaurant

Minuit était le moment qu'il attendait pour aller au restaurant

\section{Compléments de Cause}

Certains relateurs de cause ont une syntaxe complexe mettant en jeu des restructurations. C'est le cas de la racine caus-, que l'on trouve dans la locution conjonctive à cause de : Un accident grave s'est produit à cause de la pluie. Ce complément est mobile mais son statut ne change pas pour autant : À cause de la pluie, un grave accident s'est produit. En revanche, en inversant les arguments, on permet au substantif de devenir sujet :

La pluie a (produit, provoqué) un grave accident 
Ces transformations sont très générales et ne mettent pas en jeu des lexèmes spécifiques permettant le changement de structure. D'autres constructions ont cette possibilité. Certaines constructions causales construites autour de la préposition pour sont constituées d'une principale comportant un verbe de « sanction » comme punir ou récompenser et d'une subordonnée avec un verbe désignant une action considérée comme condamnable ou louable :

On a condamné Paul à dix ans de prison pour ce meurtre

On a attribué le prix Nobel à Paul pour ce roman

Un des emplois du verbe valoir permet aux compléments de cause de devenir sujets :

Ce meurtre a valu à Paul dix ans de prison

Ce roman a valu à Paul le prix Nobel

Dans certains contextes, valoir peut avoir comme équivalents des verbes comme : assurer, attirer, causer, coûter, faire, mériter, procurer, rapporter. De même pour peut, dans divers environnements, être remplacé par : grâce à, à cause de, à l'aide de, à la faveur de, au moyen de, avec, dans la suite, moyennant, par, par suite de.

\section{Compléments de but}

La locution dans le but de n'est pas la seule distribution du substantif but. Comme il s'agit d'un prédicat nominal, il peut être accompagné de son verbe support : Paul s'est levé dans le but de protester ; Paul s'est levé, il avait pour but de protester. Cette dernière phrase peut faire l'objet d'un certain nombre de restructurations qui mettent l'accent respectivement sur le prédicat de la principale (dans la structure standard où la principale précède la subordonnée) et sur celui de la subordonnée :

Paul s'est levé, il avait pour but de protester

Protester était le but qu'avait Paul en se levant

Mais on peut aussi mettre l'accent sur le relateur de finalité (but) :

Le but qu'avait Paul en se levant était de protester 
La notion de finalité peut être prise en charge par un substantif comme idée :

Paul est parti en Amérique dans l'idée de faire fortune

L'idée de faire fortune l'a poussé à partir en Amérique

\section{Compléments de CONdition}

Des opérations de restructurations analogues peuvent concerner certains compléments conditionnels :

Jean prendra le départ à condition que Paul lui fasse signe

Une inversion des arguments se fait grâce au verbe conditionner ou à son équivalent nominal condition :

Que Paul lui fasse signe conditionne son départ

Un signe de la part de Paul est la condition de son départ

La condition de son départ est un signe de la part de Paul

Cette relation peut être prise en charge par des éléments lexicaux comme : à condition de, déterminer, dicter, influencer.

\section{Compléments de CONCESSION}

Le Dictionnaire de linguistique de Dubois et alii (Larousse) définit ainsi la concession :

Une proposition subordonnée est dite concessive quand elle indique la raison qui pourrait s'opposer à l'action indiquée par la principale; elle est introduite par les conjonctions ou locutions conjonctives bien que, quoique, malgré que, encore que, même si, quelque... que, si... que, tout... que, etc. Bien que la séance fût terminée, de petits groupes restaient dans la salle.

Il existe des verbes traduisant une opposition et permettant à un complément concessif de passer en position de sujet :

Paul est parti malgré la pluie

La pluie n'a pas empêché le départ de Paul 
La notion d'opposition peut être prise en charge par des verbes comme : empêcher, interdire ou des substantifs comme obstacle, empêchement :

Il est parti malgré la pluie

La pluie ne l'a pas empêché de partir

Il ment nonobstant ses fonctions ministérielles

Ses fonctions ministérielles ne lui interdisent pas de mentir

Il a pris la parole en dépit de la peur qui le paralysait

La peur qui le paralysait n'était pas un obstacle à sa prise de parole

On peut considérer comme substantifs d'empêchement des noms comme : barrière, contretemps, difficulté, embarras, entrave, frein, gêne, obstacle, opposition, dont des antonymes seraient : aide, autorisation, encouragement, facilité, permission. Parmi les verbes exprimant un empêchement, on peut noter : bannir, censurer, condamner, défendre, empêcher, exclure, faire obstacle, fermer, inhiber, prohiber, proscrire, s'opposer, supprimer, suspendre. Des antonymes de ces verbes seraient : accorder, autoriser, permettre, tolérer.

\section{Constructions qualitatives}

Avec les prédicats nominaux désignant des qualités ou des défauts, on observe la possibilité d'avoir une construction en il y a, suivie d'un substantif humain en fonction de complément locatif :

Il y a chez Paul une grande volonté de nuire

Le verbe avoir permet à ces humains « locatifs » de devenir sujets :

Paul a une grande volonté de nuire

Voici deux autres exemples :

Il y a beaucoup de défauts chez cet enfant

Cet enfant a beaucoup de défauts

Il y a dans cette assemblée tous les défauts de la terre

Cette assemblée concentre tous les défauts de la terre 


\section{CONCLuSION}

Toutes les analyses que nous avons proposées mettent en évidence le fait qu'une description purement structurale, c'est-à-dire statique, ne permet pas de rendre compte du comportement des arguments par rapport au prédicat et de la relation qu'entretiennent les compléments " circonstanciels » avec le reste de la phrase. Nous avons vu que des phénomènes pragmatiques comme la thématisation doivent faire partie intégrante de la description linguistique, si l'on veut rendre compte du comportement réel des unités grammaticales. Faute d'analyses empiriques suffisantes sur la base d'un très grand nombre de propriétés, les compléments circonstanciels sont encore à l'heure actuelle un domaine négligé de la grammaire.

\section{Références bibliographiques}

ANSCOMBRE, Jean-Claude (1984), « La représentation de la notion de cause dans la langue », Cahiers de grammaire, $\mathrm{n}^{\circ}$ 8, pp. 3-53.

CADIOT, Pierre (1990), " À propos du complément circonstanciel de but », Langue française, $\mathrm{n}^{\circ} 86$, pp. 51-64.

CADIOT, Pierre (1991), De la grammaire à la cognition : la préposition pour, Paris, Éditions du CNRS.

DELBECQUE, Nicole (1994), «Por et/ou para : des relations entre causalité et finalité dans la phrase espagnole », Revue de linguistique romane, vol. 58, pp. 367-385.

DUBOIS, Jean et al. (1973), Dictionnaire de linguistique et des sciences du langage, Paris, Librairie Larousse.

GINGRAS, Line (1991), " Dans le but de, dans un but de, dans un but (+adj) », L'actualité terminologique, vol. $24, \mathrm{n}^{\circ} 1$, p. 4.

GIRY-SCHNEIDER, Jacqueline (1987), Les prédicats nominaux en français : les phrases simples à verbes supports, Genève, Droz.

GROSS, Gaston (1988), "Réflexions sur la notion de locution conjonctive », Langue française, $\mathrm{n}^{\circ} 77, \mathrm{pp} .19-36$.

GROSS, Gaston (1993), « Trois applications de la notion de verbe support », L'information grammaticale, $\mathrm{n}^{\circ} 59$, pp. 16-22.

GROSS, Gaston (1994), "Connecteurs et traitement automatique », in TA-TAO : recherches de pointe et applications immédiates (A. Clas, P. Bouillon éds), Beyrouth, F.M.A./Montréal, AUPELF-UREF, pp. 287-306.

GROSS, Gaston (1996) «Une typologie sémantique des connecteurs : l'exemple de la cause », Studi italiani di linguistica teorica e applicata, vol. XXV, $\mathrm{n}^{\circ} 1$, pp. 153-179.

GROSS, Gaston (1996), « Semantische Umgebung der Konnektoren », Leuvense Bijdragen, $\mathrm{n}^{\circ}$ 84, pp. 295-311. 
GROSS, Gaston (1999), « Élaboration d'un dictionnaire électronique », Bulletin de la Société de Linguistique de Paris, t. XCIV, fasc. 1, pp. 113-138.

GROSS, Gaston (1999), «Étude lexicale des locutions conjonctives », Mémoires de la Société de Linguistique de Paris, Nouvelle Série, t. VII, pp. 23-40.

GROSS, Gaston (1999), « Sémantique lexicale et connecteurs », Langages, n 136, pp. 76-84.

GROSS, Gaston (2005), «Les circonstancielles sont des complétives », in La syntaxe au cour de la grammaire. Recueil offert en hommage pour le 60 anniversaire de Claude Muller (F. Lambert, H. Nølke éds), Rennes, Presses Universitaires de Rennes, pp. 121-127.

GROSS, Gaston, PRANDI, Michele (2004), La Finalité. Fondements conceptuels et genèse linguistique, Bruxelles, De Boeck-Duculot.

GROSS, Maurice (1981), «Les bases empiriques de la notion de prédicat sémantique », Langages, $\mathrm{n}^{\circ} 63$, pp. 7-52.

GROSS, Maurice (1986), Grammaire transformationnelle du français. Syntaxe de l'adverbe, Paris, Asstril.

GUIMIER, Claude et al. (1993), 1001 Circonstants, Caen, Presses Universitaires de Caen.

HARRIS, Zellig S. (1976), Notes du cours de syntaxe, Paris, Le Seuil.

HWANG, ShinJa, MELBY, Alan (1997), « Purpose clauses in English and Korean », The LACUS forum, $\mathrm{n}^{\circ} 23$, pp. 495-508.

KUPPEVELT, Jan van (1996), « Directionality in discourse: Prominence differences in subordination relations », Journal of Semantics, vol. 13, n 4, pp. 363-395.

LA FAUCI, Nunzio (1988), « Le sujet des conjonctions de subordination finales », Langue française, $\mathrm{n}^{\circ} 77$, pp. 37-46.

LEEMAN, Danielle (1995), « Pourquoi peut-on dire Max est en colère mais non *Max est en peur ? Hypothèses sur la construction être en $N »$, Langue française, ${ }^{\circ} 105$, pp. 55-69.

MEL'ČUK, Igor (1988), « Principes et critères de description dans le DEC », in DEC 2 (I. Mel'čuk et al. éds), Montréal, Les Presses de l'Université de Montréal, pp. 27-39.

MULLER, Claude (1996), La subordination française, Paris, Armand Colin.

PIOT, Mireille (1996), Composition transformationnelle des phrases par subordination et coordination, thèse de doctorat, Université Paris 7.

POLENZ, Peter von (1963), Funktionsverben im heutigen Deutsch (Wirkendes Wort, Beiheft 5), Düsseldorf, Schwann.

PRANDI, Michele (1995), «Le proposizioni finali in italiano : uno studio di grammatica filosofica », Cuadernos de Filología Italiana, ${ }^{\circ}$ 2, pp. 45-73.

RIEGEL, Martin, PELLAT, Jean-Christophe, RIOUL, René (1994), Grammaire méthodique du français, Paris, PUF.

ZEMB, Jean-Marie (1993) « Durch was und wozu wird im deutschen Satz welches Vorfeld besetzt? », Sprachwissenschaft, vol. 18, n 1, pp. 1-28. 